\title{
Time Course of Atrial Fibrillation-induced Cellular Structural Remodeling in Atria of the Goat
}

\author{
Jannie Ausma ${ }^{1}$, Nicolle Litjens ${ }^{1}$, Marie-Hélène Lenders ${ }^{1}$, Hans Duimel ${ }^{1}$, \\ Frans Mast ${ }^{1}$, Luc Wouters ${ }^{2}$, Frans Ramaekers ${ }^{1}$, Maurits Allessie ${ }^{1}$ and \\ Marcel Borgers ${ }^{1,2}$ \\ ${ }^{1}$ Cardiovascular Research Institute Maastricht, The Netherlands and ${ }^{2} J a n s s e n$ Research Foundation, \\ Beerse, Belgium
}

(Received 5 June 2001, accepted for publication 5 September 2001)

\begin{abstract}
J. Ausma, N. Litjens, M.-H. Lenders, H. Duimel, F. Mast, L. Wouters, F. Ramaekers, M. Allessie and M. Borgers. Time Course of Atrial Fibrillation-induced Cellular Structural Remodeling in Atria of the Goat. Journal of Molecular and Cellular Cardiology (2001) 33, 2083-2094.

Background: Previously we documented cellular structural changes of a non-degenerative nature in atrial myocytes after atrial fibrillation (AF) in the goat. The time course of these changes was not studied.

Methods and Results: Cellular structural changes were studied by light- and electron microscopy and immunohistochemistry in goat atria after 0-16 weeks AF. The first sign of cellular structural remodeling was a more homogeneous chromatin distribution, at 1 week of AF. Sub-structural changes in mitochondria and sarcoplasmic reticulum occurred gradually. Cellular degeneration was absent. The degree of myolysis and glycogen accumulation increased till 8 weeks of AF and did not increase further from thereon. After 16 weeks of AF, $42 \%$ of the myocytes in the right atrial free wall were affected by myolysis. The diameter of the atrial myocytes increased. Dedifferentiation of the atrial myocytes was suggested by altered expression patterns of structural proteins, such as the disappearance of cardiotin (1 week), the A-I junctional part of titin (4 weeks), desmin at the intercalated disk (ID) (8 weeks) and a gradual re-expression of $\alpha$-smooth muscle actin.

Conclusion: Remodeling of the cellular ultrastructure in atrial myocardium of the goat develops progressively during AF. Re-expression of fetal proteins indicate dedifferentiation of atrial myocytes, analogous to observations in hibernating myocardium of the ventricle.

(c) 2001 Academic Press
\end{abstract}

KeY WoRDs: Atrial fibrillation; Ultrastructure; Histology; Remodeling; Dedifferentiation.

\section{Introduction}

Atrial fibrillation $(\mathrm{AF})$ is the most common arrhythmia in patients; paroxysmal AF often progresses to sustained AF. ${ }^{1}$ After cardioversion the electrical and contractile functions of the atria are impaired and recurrences of $\mathrm{AF}$ are frequent. Apart from increasingly severe changes due to an underlying heart disease, AF itself causes progressive electrophysiological changes. In humans, subsequent to electrical remodeling, ultrastructural changes of a degenerative nature have been described in atrial myocytes after long-term lone $\mathrm{AF}^{2}$ and $\mathrm{AF}$ in combination with other etiologies. ${ }^{3-5}$ It has been established that chronic rapid atrial pacing in dogs and repeated induction of $\mathrm{AF}$ in goats results in the development of sustained AF (AF begets AF) ${ }^{6,7}$ This increasing stability can only partly be explained by the occurrence of electrical remodeling (shortening of the atrial effective refractory period) during the first 2 days of $\mathrm{AF}^{7}$ Since AF becomes sustained after 1-2 weeks, a

Please address all correspondence to: Dr J. Ausma, Department of Physiology, Cardiovascular Research Institute Maastricht, P.O. Box 616, 6200 MD Maastricht, The Netherlands. Tel: + 31-43-3881076; Fax: + 31-43-3884166; E-mail: jannie.ausma@fys.unimaas.nl 
second factor with a slower time course must be involved.

Previously, we have described a variety of cellular structural changes in goat atrial myocytes after months of experimentally induced AF, with depletion of contractile elements and accumulation of glycogen as main features. These changes were accompanied by a two-fold increase in the myocyte cell size. Furthermore, there were no signs of cellular degeneration (necrosis or apoptosis) and the interstitial space remained unaltered. ${ }^{8-10}$ Using immunohistochemistry the expression and organization of structural proteins were assessed. The re-expression of $\alpha$-smooth muscle actin, the disappearance of cardiotin and the staining patterns of titin and desmin were reminiscent of fetal myocytes, indicating that chronic AF induces myocyte dedifferentiation. ${ }^{10}$

The chronic AF goat model is highly suitable to study structural remodeling due to AF. Till now it was unknown when the cellular structural changes occur after the induction of AF and how the severity of alterations progresses in time. Therefore, the time course of cellular structural changes has been studied during 16 weeks of AF in goats at several atrial sites by light- and electron microscopy and immunohistochemistry.

\section{Material and Methods}

\section{The goat model of chronic AF}

The goat model of AF as described previously was used. $^{6}$ Under anesthesia an Itrel-pacemaker (Medtronic $^{\circledR}$ Bakken Research Center, Maastricht, The Netherlands) was implanted in the neck of the goat and a bipolar screw-in electrode was inserted through the jugular vein in the right atrium. Atrial fibrillation was maintained by burst stimulation as described previously. ${ }^{11}$ Thirty-six goats $(61 \pm 13 \mathrm{~kg})$ were used (six groups of six animals) in this study. The control group was kept in sinus rhythm (SR), whereas in the other groups AF was maintained for 1, 2, 4, 8 and 16 weeks respectively. Animal handling was carried out according to the Dutch Law on Animal Experimentation (WOD) and The European Directive for Protection of Vertebrate Animals Used for Experimental and Other Scientific Purposes.

\section{Tissue processing}

At the end of the experimental period, the goats were anaesthetized and thoracotomy was performed. The right and left atrial appendages were excised and immediately frozen in liquid nitrogenprecooled isopentane. These frozen samples were used for immunohistochemistry. The heart was excised and fixed by retrograde perfusion with 3\% glutaraldehyde in $90 \mathrm{~mm} \mathrm{KH}_{2} \mathrm{PO}_{4}$ buffer $(\mathrm{pH} 7.4){ }^{11}$ For the immunohistochemical analysis of $\alpha$-smooth muscle actin, left and right atrial samples were stored after $15 \mathrm{~min}$ fixation in $\mathrm{KH}_{2} \mathrm{PO}_{4}$ buffer at $4^{\circ} \mathrm{C} .{ }^{10}$ For light- and electron microscopic studies, eight atrial parts [right atrial free wall (RAFW), right trabeculae (RT), right atrial appendage (RAA), left atrial free wall (LAFW), left trabeculae (LT), left atrial appendage (LAA), interatrial septum (IAS) and Bachmann's bundle (BB)] were divided into blocks $\left(4 \mathrm{~mm}^{3}\right)$ and kept for at least $24 \mathrm{~h}$ in glutaraldehyde. The fixed blocks were washed with $\mathrm{KH}_{2} \mathrm{PO}_{4}$ buffer containing $7.5 \%$ sucrose, postfixed with $2 \% \mathrm{OsO}_{4}$ in $50 \mathrm{~mm}$ veronal acetate buffer for $1 \mathrm{~h}$, dehydrated through graded ethanol series and routinely embedded in epoxy resin Epon. ${ }^{8}$ For electron microscopy, ultrathin sections cut from each Epon sample, were counterstained with uranium acetate and lead citrate. ${ }^{11}$ The sections were examined with a Philips CM100 microscope.

\section{Light microscopic quantification of atrial remodeling}

\section{Myolysis}

Sections of $2 \mu \mathrm{m}$ from the eight different atrial areas were examined by light microscopy. To quantify the degree of myolysis and glycogen accumulation, sections were stained with periodic acid Schiff (PAS) and toluidine blue. ${ }^{8}$ At least three sections per atrial site were examined with a minimum of 300 cells in total. Only myocytes in which the nucleus was present in the plane of the section were evaluated. Myocytes were considered mildly myolytic if myolysis involved 10-25\% of the cytosol, and as severely myolytic when myolysis involved $>25 \%$ of the cytosol. $^{8}$

\section{Cell size}

The diameter of the atrial myocytes (the shortest axis through myocytes with a nucleus in the plane of the section) was measured with a digital imaging system (Sony CCD camera $^{\circledR} /$ Macintosh $^{\circledR} / \mathrm{NIH}$ image software). Diameter measurements were performed for three categories of cells (1) normal cardiomyocytes (without myolysis), (2) mildly affected myocytes (10-25\% myolysis) and (3) severely affected myocytes ( $>25 \%$ myolysis). The measurements were performed for all eight atrial sites. The number of cells 
evaluated per site was 50 and two different areas from each site were included in the analysis.

\section{Connective tissue}

The relative amount of connective tissue in the myocardium was determined by morphometry with a grid as described previously. The total number of intersections in connective tissue was expressed as the percentage of total number of intersections. ${ }^{8}$

\section{Immunohistochemical staining procedures}

Indirect immunofluorescence assays with different primary antibodies were performed on frozen sections as described before. ${ }^{10}$ The immunoperoxidase method was only used for staining of $\alpha$-smooth muscle actin in glutaraldehyde-fixed samples. ${ }^{10}$

\section{Statistical analysis}

The effect of duration of AF on myolytic changes, cell size and connective tissue content at the different atrial sites was evaluated by regression analysis using a second degree polynomial. P-values $<0.05$ were considered as statistically significant. A mixed model analysis of co-variance (ANCOVA) was used to determine whether the effect of AF was influenced by atrial site or degree of myolysis. ${ }^{12}$ Since the large number of comparisons involved in the latter analysis only $P$-values $<0.01$ were considered as statistically significant. For statistical analysis the SAS 6.11 program was used. ${ }^{13}$

\section{Results}

\section{Time course of ultrastructural changes due to $\mathrm{AF}$}

Atrial myocytes from goats in SR show regularly distributed sarcomeres throughout the cytoplasm with rows of mitochondria in between (Fig. 1a,e). After 1 week of AF the first changes in cellular ultrastructure appeared. The majority of the nuclei redistributed heterochromatin homogeneous throughout the nucleus (Fig. 1b). After 1 and 2 weeks of AF myolysis and glycogen accumulation occurred to a small extent in part of the atrial myocytes and mitochondria retained their normal shape (Fig. 1f). In the weeks following these initial changes there was a gradual increase in the number and area of the cells in which myolysis and glycogen accumulation became manifest (see quantitative data below) [Figs 1(c,d) and 2]. In areas with depleted sarcomeres mitochondria became elongated, showing reorientation of cristae in longitudinal arrays (Fig. 1g,h). Disorganized membranes, representing sarcoplasmic reticulum remnants, were present in the myolytic cytosol in part of the atrial myocytes (Fig. 1g). The depletion of sarcomeres did not result in atrophy; instead the cardiomyocytes were enlarged.

\section{Quantification of the changes in cellular structure by light microscopy}

\section{Quantification of myolysis}

In goats with sustained AF, the number of atrial myocytes with myolytic changes $(>10 \%$ of the cell cytosol surface free from sarcomeres) and glycogen accumulation increased in time at different atrial sites (Table 1; Fig. 3a,b). For all atrial sites apart from $\mathrm{BB}(P=0.072)$, there was a highly significant increase $(P=0.001)$ in the number of cells affected by myolysis. At some of the atrial sites (RAFW, RAA, RT, LAA) the linear increase in number of atrial myocytes affected by myolysis reached a maximum after about 8 weeks as indicated by the significance of the quadratic term of the polynomial model. The average number of myolytic cells was higher in the right than in the corresponding left atrial sites. The number of myolytic cells was most pronounced in RAA followed by RAFW and RT. At the left atrial sites the same order in the severity of the changes appeared, LAA, followed by LAFW and LT. The lowest numbers of cells affected by myolysis were present in BB and IAS. Mixed model ANCOVA followed by multiple comparison showed that the linear dependency of the number of myolytic cells as a function of the duration of $\mathrm{AF}$ was more significant for RAA than for IAS, LT, LAFW and BB $(P<0.01)$. For LAA only the IAS had a significantly lower number of affected cells $(P<0.01)$.

Similar results were found in relation to $\mathrm{AF}$ duration when only the severely affected cells (>25\% myolysis) were taken into account (Table $1)$. The percentage of cells with severe myolysis increased significantly $(P<0.05)$ with the duration of $\mathrm{AF}$ at all atrial sites, with the exception of $\mathrm{BB}$ $(P=0.179)$. Again, in the right atrial sites the changes were more pronounced than in their left atrial counterparts.

\section{Quantification of atrial cell size}

The diameters (shortest axis) of myocytes increased significantly $(P<0.05)$ at all atrial sites, being most 

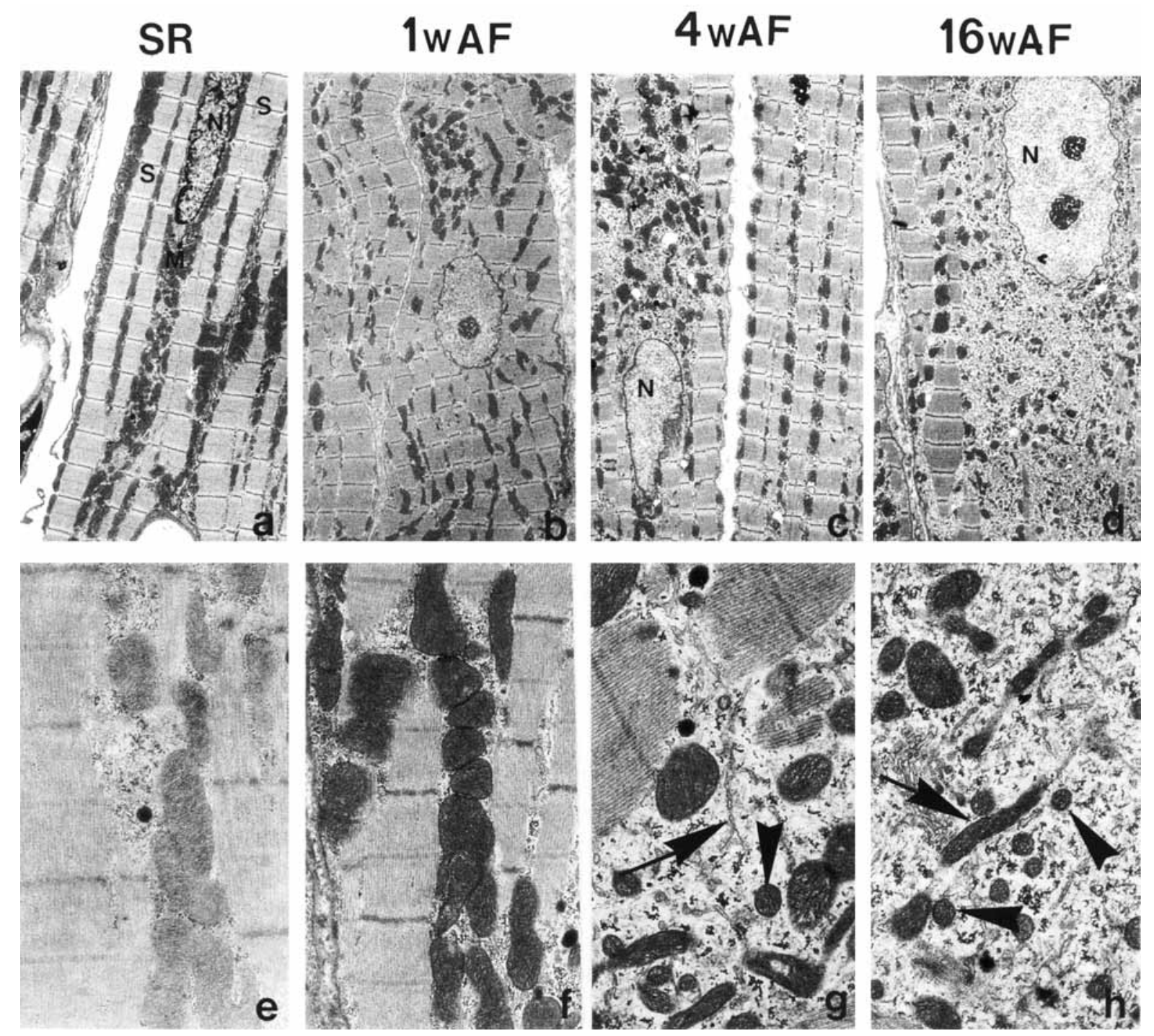

Figure 1 Electron microscopic pictures of goat atrial myocardium. (a) Atrial myocardium during SR. Sarcomeres (s) are present throughout the entire cytosol with rows of mitochondria $(\mathrm{m})$ in between. The nucleus $(\mathrm{n})$ shows perinuclear heterochromatin clustering $(\times 3200)$. (b) After 1 week of AF, myocytes show the first cellular change: a homogeneous distribution of nuclear heterochromatin $(\times 2400)$. (c) Myolysis and glycogen accumulation after 4 weeks of AF. The nuclear $(\mathrm{n})$ area shows less clustering of heterochromatin. The neighbouring myocyte (right) still has a normal organization with rows of mitochondria between the sarcomeres $(\times 2400)$. (d) Severely affected cardiomyocytes after 16 weeks of AF. Accumulation of glycogen $(\mathrm{g})$ in the central part of the cardiomyocytes. Sarcomeres are depleted and numerous abnormally shaped small mitochondria are present. The nucleus (n) shows a homogeneous distribution of nuclear heterochromatin $(\times 2200)$. (e) Detail of normal mitochondria of atrial myocardium in SR $(\times 16000)$. (f) Mitochondria between the sarcomeres have a normal appearance after 1 week of AF $(\times 13000)$. $(\mathrm{g})$ Smaller mitochondria (arrowhead) appear in the myolytic areas after 4 weeks of AF. Remnants of sarcoplasmic reticulum (arrow) at the border of an area devoid of sarcomeres $(\times 16000)$. (h) Slender elongated mitochondria with longitudinally oriented cristae (arrow), also displayed as small round structures when cross sectioned (arrowhead), present in myolytic areas which are mainly filled with glycogen after 16 weeks of AF $(\times 16000)$.

pronounced in LAFW and LAA. At the LAFW the average atrial myocyte diameter increased from $12.3 \mu \mathrm{m}$ during SR to $21.2 \mu \mathrm{m}$ after 16 weeks of AF (Table 1 and Fig. 3c,d). Interestingly, when only non-myolytic cells were considered, the increase in cell diameter in relation with AF duration could only be observed in the RAFW, RT, LAFW and
BB (Table 1). At the LAFW, the average diameter increased from $12.3 \mu \mathrm{m}$ during SR to $19.2 \mu \mathrm{m}$ after 16 weeks of AF.

Mixed model ANCOVA comparison of the linear dependency of showed that the increase in myocyte diameter for the total myocyte population was most pronounced at LAFW $(P<0.001$, compared to all 

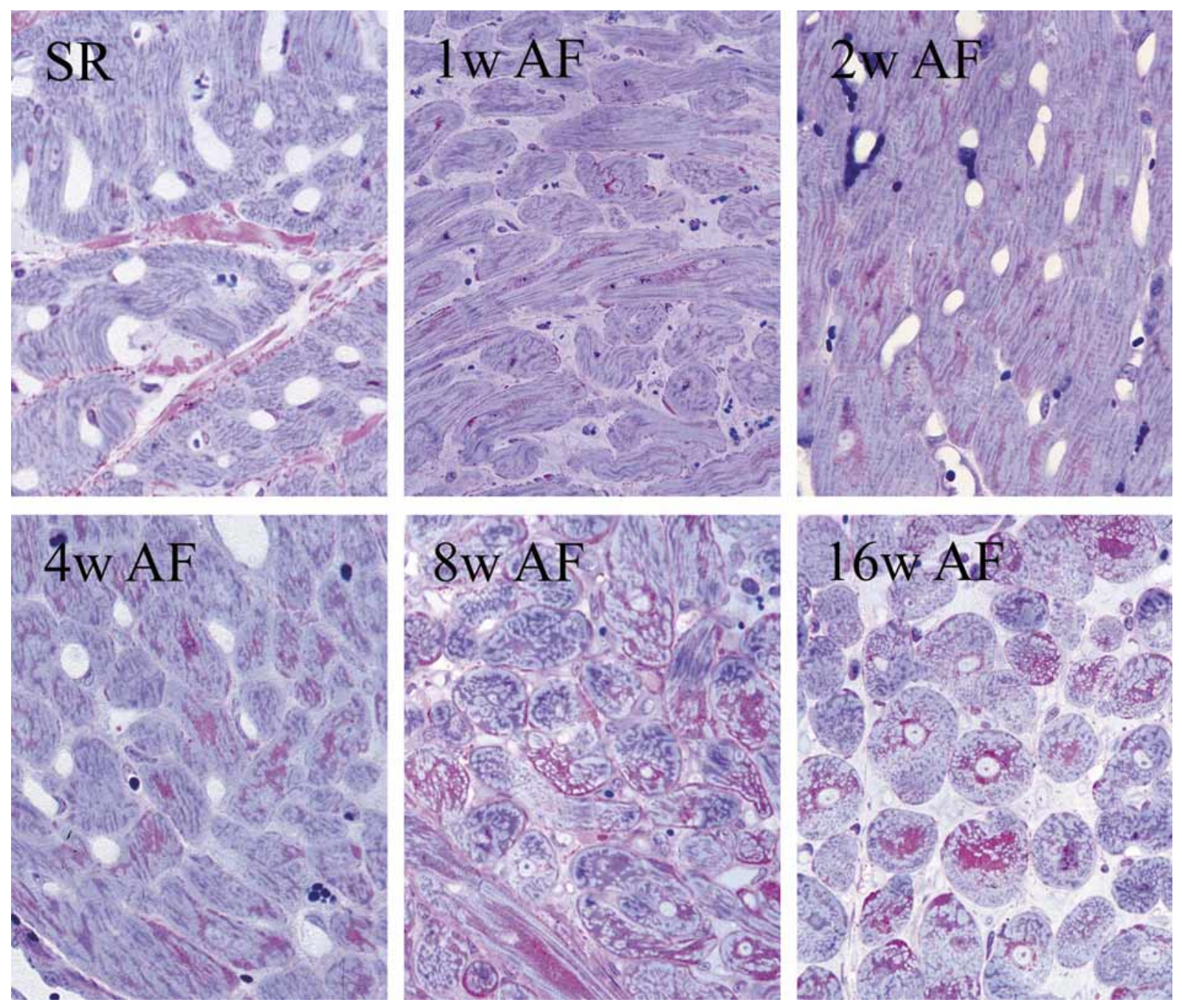

Figure 2 Light microscopic pictures of $2 \mu \mathrm{m}$ sections from goat atrial myocardium stained with periodic acid Schiff (PAS; red glycogen staining) and toluidine blue (sarcomere staining). In SR hearts, PAS positive material is virtually absent in the atrial myocyte cytosol. The sarcomeres stained with toluidine blue are present throughout the cytosol. After 1 week AF, myolysis and glycogen accumulation occur in a small number of the atrial myocytes. An increase in the number of atrial myocytes with myolysis and glycogen accumulation occurs after longer AF durations. Also the degree of myolysis and glycogen accumulation in individual cells increases with the duration of AF. Enlargement of atrial myocytes due to AF is visible $(\times 180)$.

other sites) and indicated that the atrial cell diameter depends on the extent of myolysis (nonmyolytic cells, $v$ cells with a mild degree of myolysis, $v$ cells with a severe degree of myolysis) for all atrial regions $(P<0.001$, except for $\mathrm{BB} P=0.024)$.

\section{Connective tissue}

Compared to SR, the connective tissue content did not undergo any obvious quantitative modification during 1 to 16 weeks of AF (Table 1). Infarctions were not observed and cardiomyocyte degeneration was not detected. Immunohistochemistry did not show the presence of markers for extracellular matrix remodeling (tenascin and cellular fibronectin). A normal amount of vimentin-positive mesenchymal cells were present at the different time points after induction of AF.

\section{Time course of changes in structural proteins}

\section{Cardiotin}

In goats with $S R$, the monoclonal antibody $\mathrm{R}_{2} \mathrm{G}$ visualizes staining of the structural protein cardiotin as longitudinal fibers within the myocytes. After 1 week of AF, the cardiotin staining was altered in most of the atrial myocytes. The longitudinal cardiotin arrays were shorter and stained more 


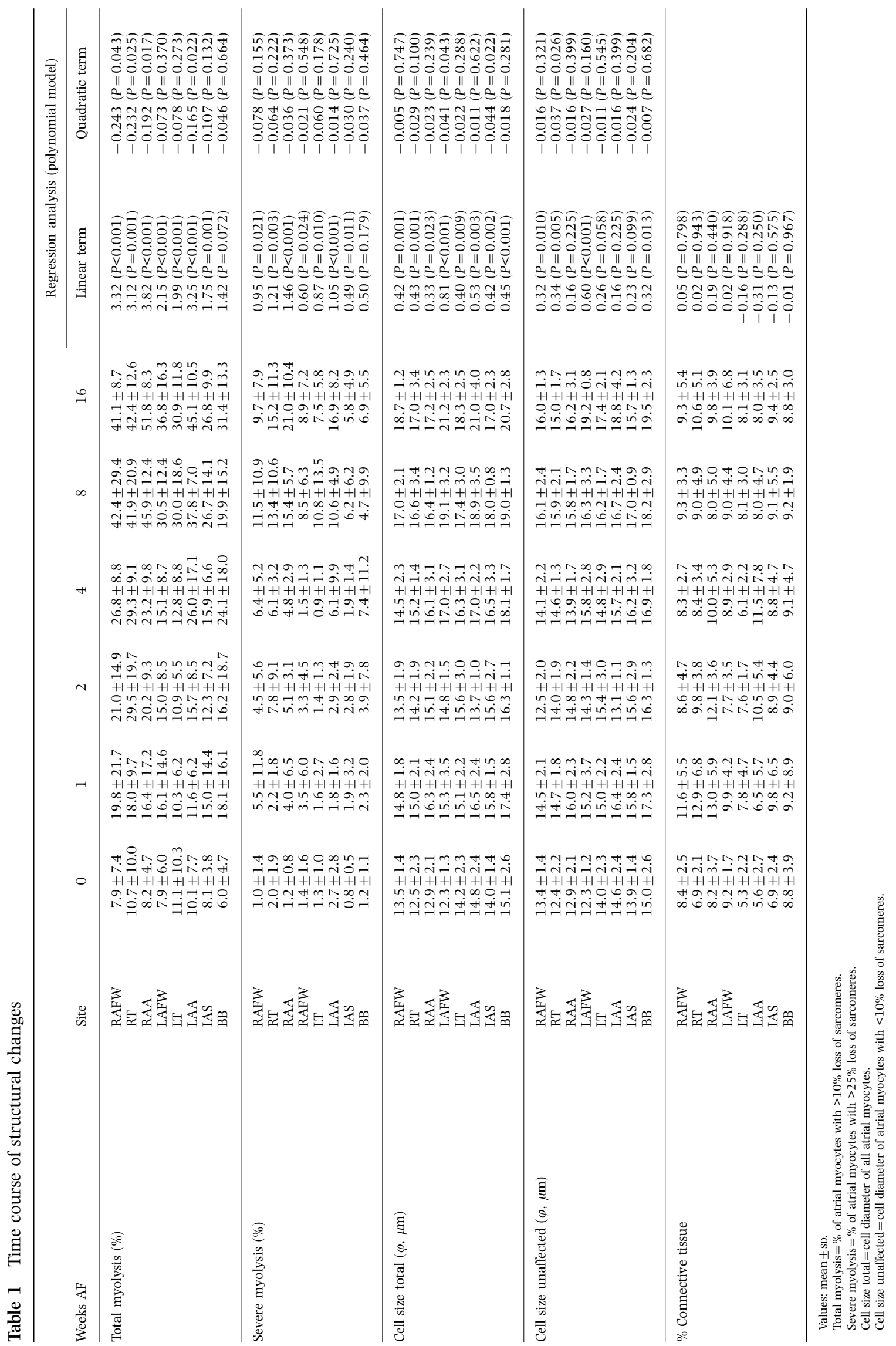




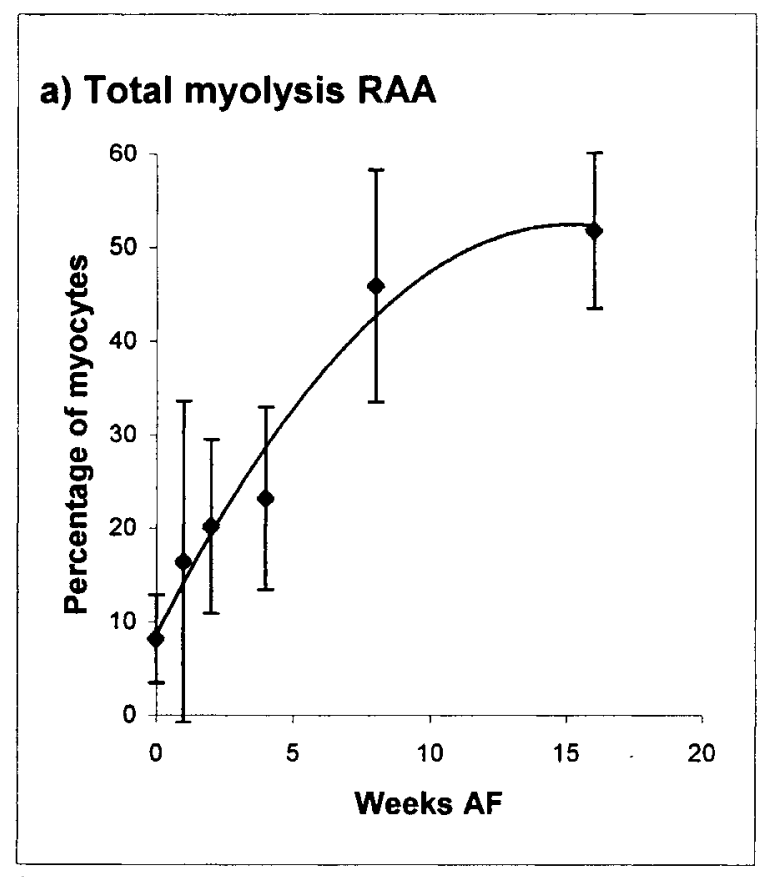

\section{b) Total myolysis IAS}

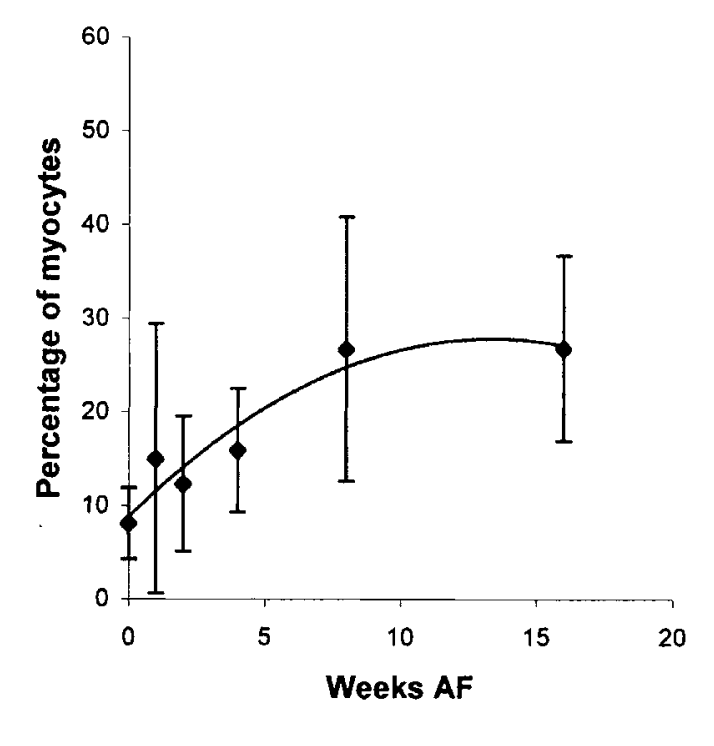

\section{c) Cell diameter LAFW}

\section{d) Cell diameter IAS}
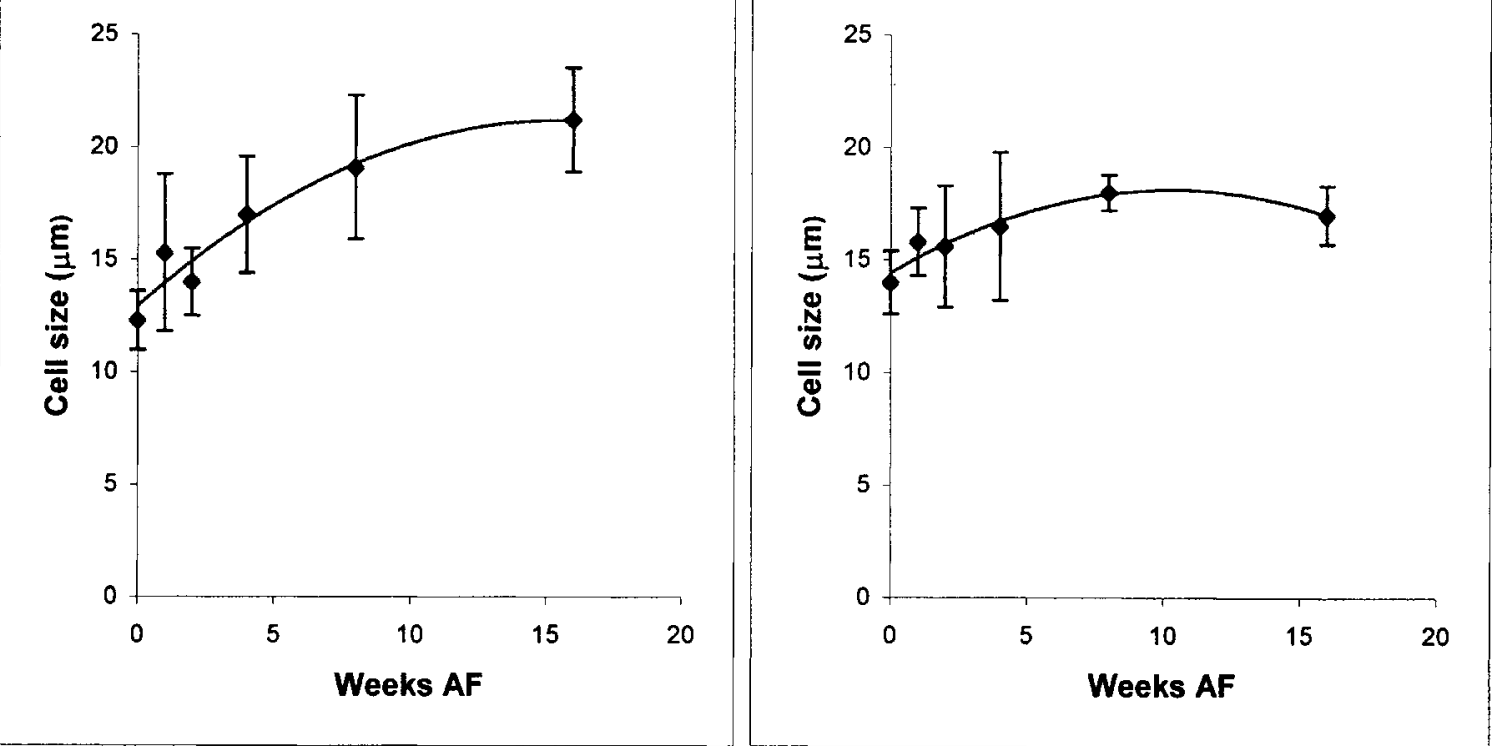

Figure $3(a, b) \quad$ Percentage of cells affected by $>10 \%$ myolysis (mild + severe) as a function of AF duration (means \pm SD). For all regions, the number of affected myocytes increases with the duration of AF. As an example the sites with the strongest response the RAA (a) and the area with the lowest response the IAS (b) are given. Lines indicate the best fitted second degree polynomial. (c,d) Cell diameters ( $\mu$ m shortest axis) (means $\pm \mathrm{sD}$ ) of atrial myocytes irrespective of the degree of myolysis (myolytic + non-myolytic), in function of AF duration. As an example, LAFW is given (c) as the site with the strongest increase in cell size and the IAS as the site with the smallest increase (d). For all regions, the atrial myocyte diameter increases with the duration of AF. Lines represent the best fitted second degree polynomials.

diffusely. From 4 weeks of AF onwards, the longitudinal cardiotin staining was absent and only a diffuse staining or no staining at all could be seen in the atrial myocytes (Fig. 4a and Table 2).

\section{Titin and its associated proteins}

Monoclonal antibodies directed against different epitopes of titin, as well as those directed to the titin-associated proteins (myomesin, M-protein and 
SR 1W AF 2W AF 4w AF 8W AF $16 \mathrm{~W} \mathrm{AF}$

a) Cardiotin
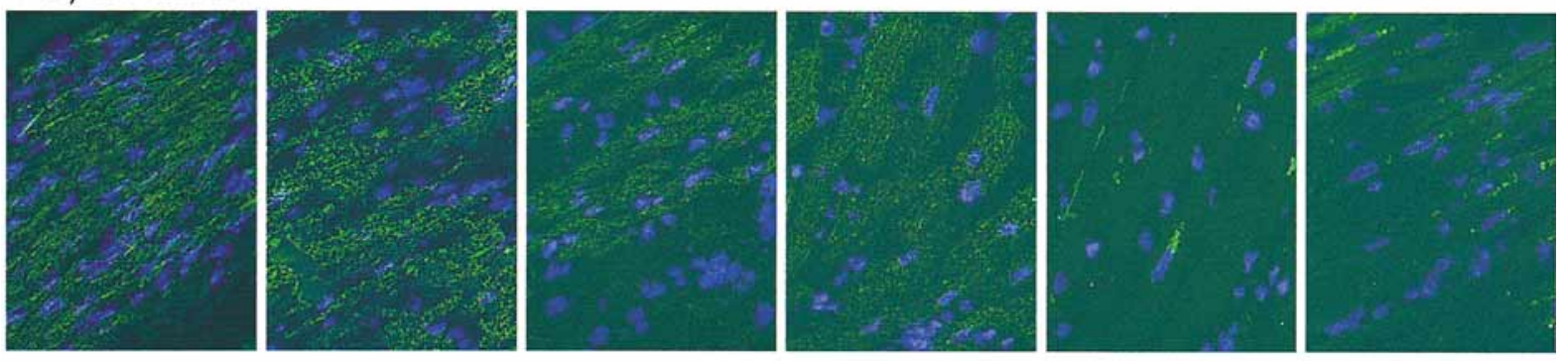

b) Titin at the A-I junction
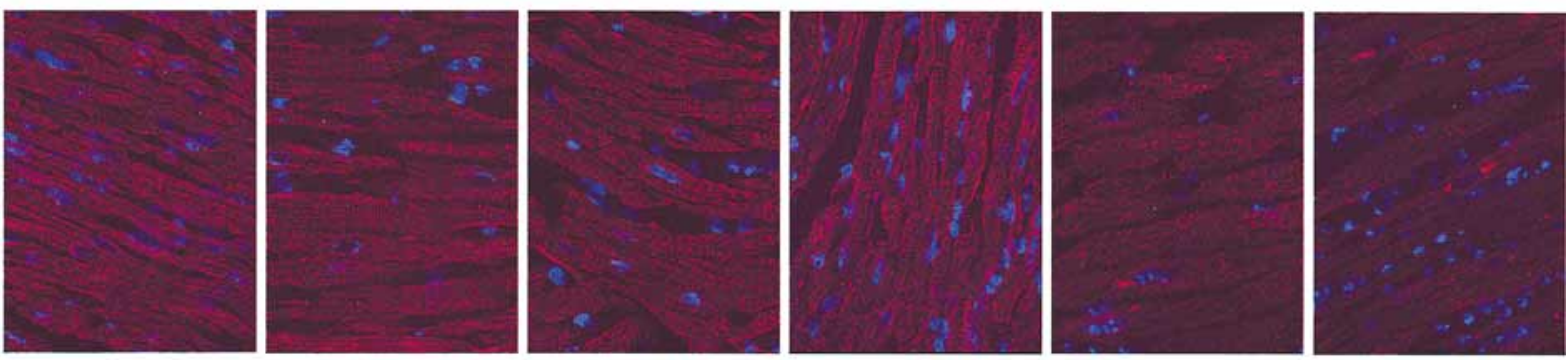

\section{c) $\alpha$-smooth muscle actin}

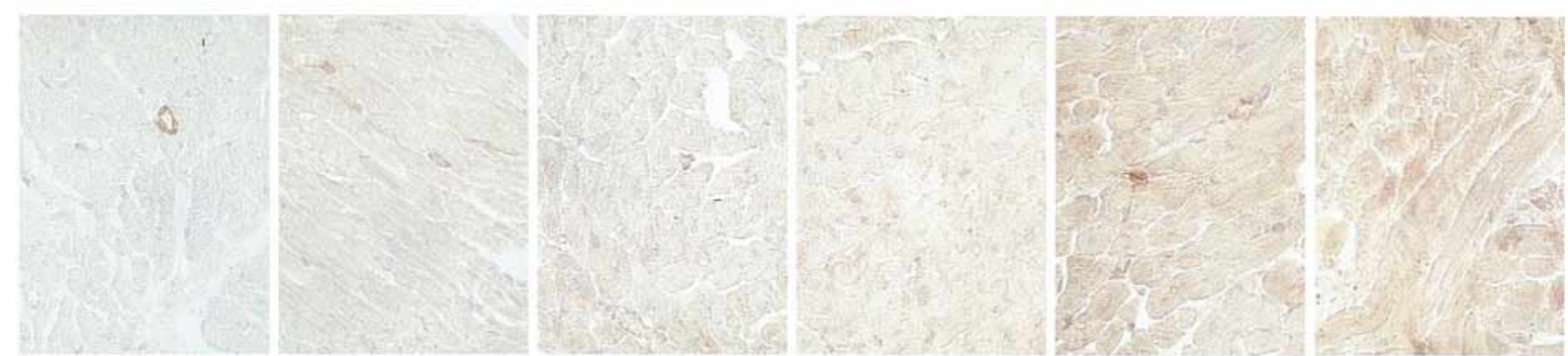

\section{d) Desmin at the intercalated disk}
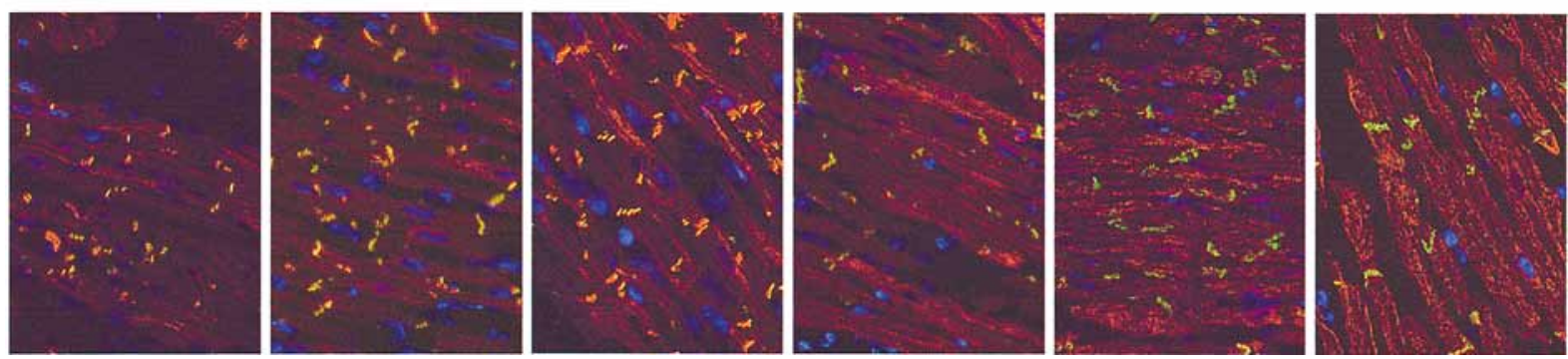

Figure 4 Immunohistochemistry of structural proteins on sections from goat atrial myocardium in SR and after 1, 2, 4,8 and 16 weeks of AF. (a) Immunofluorescence staining with anti-cardiotin (green) and DAPI nuclear staining (blue). Cardiotin, displayed as longitudinally orientated filaments during SR, loses its regular organization after 1 week of AF and is absent from 4 weeks of AF onwards. (b) Immunofluorescence with 9D10 antibody directed against the A-I junctional part of titin (red) and DAPI-nuclear staining (blue). Normal cross-striated patterns are present during SR and after 1 and 2 weeks AF. After 4 weeks, the intense titin staining is lost and sometimes only punctated staining of titin remains. After 16 weeks of AF, only residual titin staining is visible. (c) Immunoperoxidase labeling of $\alpha$-smooth muscle actin (red-brown). $\alpha$-Smooth muscle actin in SR goats is absent from atrial myocytes, only vascular smooth muscle cells are stained. After 1 week of $\mathrm{AF}, \alpha$-smooth muscle actin is visible as a diffuse weak staining which increases 
Table 2 Time course of changes in structural proteins

\begin{tabular}{llllllll}
\hline \multirow{2}{*}{ Protein } & Site & \multicolumn{7}{c}{ Weeks AF } \\
\cline { 3 - 7 } & & 0 & 1 & 2 & 4 & 8 & 16 \\
\hline \multirow{2}{*}{ Cardiotin } & RAA & + & \pm & \pm & - & - & - \\
\multirow{3}{*}{ Titin } & LAA & + & \pm & \pm & - & - & - \\
\multirow{3}{*}{ Smooth muscle actin } & RAA & + & + & + & \pm & \pm & - \\
\multirow{5}{*}{ Desmin at ID } & LAA & + & + & + & \pm & \pm & - \\
& RAA & - & \pm & \pm & \pm & + & + \\
& LAA & - & \pm & \pm & \pm & + & + \\
& RAA & + & + & + & \pm & - & - \\
& LAA & + & + & + & \pm & - & - \\
\hline
\end{tabular}

$+=$ present, $-=$ absent.

C-protein) showed clear cross-striated staining patterns during SR in goats. Similar staining patterns were observed after 1 and 2 weeks AF. After 4 weeks AF part of the atrial myocytes showed loss of the typical cross-striated staining pattern and the staining intensity was weaker with antibodies directed against the A-I junctional part of titin (9D10 and T30) (Fig. 4b and Table 2) and for Cprotein $(\mathrm{C} 1 \mathrm{C} 2)$. With increasing duration of $\mathrm{AF}$ more atrial myocytes lost the cross-striated staining patterns of titin at the A-I junction (Table 2). At 16 weeks AF titin staining was lost in almost all atrial myocytes and only a punctated staining pattern remained.

In contrast, the staining patterns of other antibodies that recognize epitopes close to the Z- and M-line [titin (T12, T31, T41), M-protein (AA241) and myomesin antibody (BB78)], were unaltered in areas with remaining sarcomeres during the studied time course of AF. Their staining patterns were cross-striated like those from other sarcomeric proteins as actin, myosin and tropomyosin.

\section{$\alpha$-Smooth muscle actin}

Atrial myocytes from goats in SR lacked $\alpha$-smooth muscle actin staining (Figure $4 \mathrm{c}$ ). After 1 and 2 weeks AF, part of the atrial myocytes displayed a diffuse staining. A gradual re-expression of $\alpha$ smooth muscle actin occurred from 2 weeks $\mathrm{AF}$ onwards (Fig. 4c and Table 2), resulting in an increased and intensified staining in the individual myocytes. After 8 and 16 weeks of AF a substantial portion of the cells displayed an intense staining, while the remaining part displayed a diffuse $\alpha$ smooth muscle actin staining. Smooth muscle cells from blood vessels reacted strongly with the anti$\alpha$-smooth muscle actin antibody throughout the whole time course.

\section{Cell adhesion molecules}

In myocytes from goats in SR and after 1, 2 and 4 weeks AF, desmin staining co-localized with the desmosomal proteins desmoplakin (Fig. 4d) and desmoglein. From 4 weeks onwards, the regular desmin cross-striation was lost. Desmin remained in the myolytic areas where sarcomeric proteins as myosin and actin were absent. After 8 weeks of AF, desmin staining was lost from the intercalated disks in a substantial portion of the atrial myocytes (Fig. 4d and Table 2) whereas desmoplakin, desmoglein and N-cadherin staining remained present. None of the intercalated disks showed desmin staining after 16 weeks of AF.

\section{Discussion}

Changes in atrial myocardium have been described for different atrial pathologies both in humans and in animal models. ${ }^{2-5,14-16}$ Structural changes

with AF duration. (d) Immunofluorescence double-labeling with desmin (green) and desmoplakin (red) and DAPInuclear staining (blue). During SR, desmin is visible in a clear cross-striated pattern. At the site of the desmosomal contact desmin staining is overlaying the desmoplakin staining (yellow staining). Desmin loses its normal regular organization in the cytoplasm of the atrial myocytes visual after 4 weeks $\mathrm{AF}$ and at 8 weeks of AF the intercalated disk shows no longer desmin staining, only desmoplakin remained (green) $(\times 250)$. 
in the atrial myocardium are thought to be a risk factor for the development of $\mathrm{AF}$ after cardiac surgery. ${ }^{14} \mathrm{~A}$ variety of changes in cellular ultrastructure occur in goats with chronic AF. ${ }^{8-10}$ However, none of the studies mentioned above examined the time course of such changes during different atrial pathologies.

\section{Quantification of the changes in cellular structure induced by $\mathrm{AF}$}

From the current study we can conclude that changes in cellular ultrastructure increase with the duration of AF. During AF, the number of myocytes affected by myolysis increased. Futhermore, the extent of myolysis within individual myocytes also increased during AF. After 8 weeks no further increase occurred at most atrial sites.

The effect of AF on the extent of myolysis appeared to be site related. Right atrial sites showed a stronger response than their left atrial counterparts. Although there is no direct evidence, it is likely that the atrial sites which are thinner have a higher propensity to undergo passive stretch, e.g. right atrial sites compared to their left atrial counterparts. The size of both myolytic and non-myolytic atrial myocytes increased with the duration of AF. It is not known whether or not the myolysis and the increase in cells size are induced independently. Both alterations occur as a result of $\mathrm{AF}$, but the factors contributing to their origin are unknown. The increase in atrial cell diameter seems to be most pronounced at the LAA and LAFW, where higher atrial pressures develop than in right atrium.

Connective tissue content did not change as a result of AF, which is indicative of the absence of overt degeneration and reactive fibrosis. This is in contrast to what has been reported in patients. ${ }^{3-5}$ This discrepancy might be due to the differences in duration of AF, species, age and/or the presence of other concomitant pathologies.

The degree of AF-induced changes in cellular ultrastructure varied among the atrial myocytes. Heterogeneity of the changes occurred not only among the different sites, as can be deduced from quantitative analysis; for an unknown reason, regional differences were also detected within one sample, i.e. unaltered atrial myocytes are intermingled with cells showing severe myolysis.

\section{Effects of changes in subcellular structure}

Redistribution of heterochromatin as seen after 1 week of AF, resembles embryonic/neonatal car- diomyocytes and is related to embryonic-like gene expression. ${ }^{17}$ After 2 weeks of AF a gradual increase of the effects on sarcomeres, glycogen, mitochondria and sarcoplasmic reticulum were seen. The impact of alterations in mitochondria is not fully understood. Enzyme cytochemical studies showed that mitochondria with an altered donut-like appearance posses normal cytochrome-C-oxidase activity. ${ }^{18}$ The loss of sarcoplasmic reticulum and contractile proteins possibly diminishes contractile force, resulting in atrial stunning and/or hibernation. If similar changes were present in man after prolonged AF, as reported by Mary-Rabine et al., ${ }^{6}$ they might explain the slow recovery (weeks to months) after cardioversion of AF in patients. ${ }^{19}$ The enhanced accumulation of glycogen in the structurally altered atrial myocytes might imply that a metabolic shift has occurred. This storage is hypothesized to sub-serve high energy demanding processes, volume regulation and mechanical cell stability. ${ }^{20}$

Changes in structural proteins: "atrial dedifferentiation"

The described cellular structural adaptations are characteristic for dedifferentiation. ${ }^{21}$ Cardiotin, a structural protein that appears late during embryonic development seems to disappear early in the reverse process during $\mathrm{AF}^{22}$ Changes in the immunohistochemical detectability of the titin epitopes at the A-I junction and the first part of the A-band after 4 weeks of AF is also suggestive of dedifferentiation. A similar staining of the A-I junctional part of titin is present during embryonic development before sarcomere formation. ${ }^{23}$ During dedifferentiation induced by AF, alterations in the scaffold protein titin precede and possibly facilitate the breakdown of other sarcomeric proteins.

The expression of $\alpha$-smooth muscle actin is seen at the onset of myofibrillogenesis. ${ }^{24}$ Re-expression of $\alpha$-smooth muscle actin during chronic AF is possibly necessary for maintaining the cell structure when the expression of other sarcomeric proteins is depressed.

During cardiogenesis, desmin is reorganized from a filamentous to a cross-striated expression pattern before co-localization with desmoplakin occurs. Therefore, loss of desmin from the intercalated disk during chronic AF resembles its organization during late stages of heart development. ${ }^{25}$ Tissue integrity is diminished due to the absence of desmin from the intercalated disk. ${ }^{26}$ 
Induction of atrial "hibernation" after prolonged AF

Ventricular myocytes of patients with low-flow ischemia (chronic hibernation) undergo a similar cellular structural adaptation as atrial myocytes during chronic AF. ${ }^{8,21}$ However, whether or not ischemia lies at the basis of the cellular remodeling during $\mathrm{AF}$, resulting in a "hibernating-like" phenotype, is not known. In dogs, oxygen consumption of the atrial tissue increases 2-3-fold after the induction of $\mathrm{AF}$, resulting in a marked reduction in the flow reserve. ${ }^{27}$ Enhanced lactate production, which is indicative of ischemia, has been found in patients with chronic $\mathrm{AF}^{28}$ However, this could not be confirmed by Lau et al. ${ }^{29}$ In dogs, atrial blood flow was reduced after 4 weeks of pacing-induced $\mathrm{AF}^{30}$ It is not known whether this reduction in blood flow is associated with atrial ischemia. ${ }^{28}$ Metabolic studies performed in pigs and goats showed that depletion of high-energy phosphates does not occur during AF. ${ }^{18,31}$ Only a reduction in phosphocreatine content was seen in both studies. The pattern of $\mathrm{Ca}^{2+}$-distribution during the first 2 weeks of $\mathrm{AF}$ resembles the distribution as seen after acute reversible ischemia. ${ }^{11}$ Taken together, these results suggest that severe ischemic damage is unlikely to occur during AF. ${ }^{11}$ On the other hand, a depressed contractile activity resulting in increased passive stretch, might influence protein expression towards dedifferentiation. ${ }^{10}$

Possible role of AF-induced cellular structural remodeling in stabilizing $\mathrm{AF}$

The chance of successful cardioversion and/or prevention of $\mathrm{AF}$ in patients is dependent on the duration of $\mathrm{AF}$ and the degree of complexity of AF. This clinically observed diminished efficacy of chemical cardioversion after long-term $\mathrm{AF}^{32}$ can not be explained solely by shortening of the atrial effective refractory period (AERP). Shortening of the AERP occurred only during the first days of AF. $^{6}$ A second factor with a slower time course might be important for the stability of $\mathrm{AF}$, since $\mathrm{AF}$ became persistent after 1-2 weeks. ${ }^{6}$ A direct link between the herein-described remodeling of the cellular ultrastructure and the electrophysiological characteristics of persistent AF has not been made. However, cellular structural remodeling, including gap-junctional remodeling, might result in inhomogeneities of the underlying atrial structure. Anisotropic conduction as a result of increasing tissue heterogeneity influences the propagation of activation front and might thereby be responsible for conduction block and reentry that stabilizes $\mathrm{AF}^{33}$ An increasing stability of AF with duration was shown in the goat by cardioversion studies. The efficacy of cardioversion by class Ic drugs was reduced from $78 \%$ after 1 month of $\mathrm{AF}$ to $30 \%$ after 4 months of $\mathrm{AF}^{34}$ Recently, another study presented evidence for the cumulative effect of prolonged AF paroxysms, which are independent of electrical remodeling, on AF stability. Goats were in AF for three consecutive 1-month periods. Following each period, the goat underwent electrical cardioversion and the AERP was allowed to return to normal values before reinduction of AF. Repetitive 1-month periods of AF promoted the stability of AF. The duration of the burst pacing required to induce sustained AF decreased after each period. ${ }^{35}$ The increasing stability of AF occurred in the same time domain (after 1 month) as the structural remodeling of the cellular structure which is suggestive of a possible relationship.

\section{Acknowledgement}

This study was supported by The Netherlands Heart Foundation (grant 96-155).

\section{References}

1. Kopecky SL, Gersh BJ, McGoon MD, Guidaudon MD, Chu CP, Ilstrup DM, Chesebro JH, Whisnant JP. The natural history of lone atrial fibrillation: A population based study over three decades. $N$ Engl J Med 1987; 317: 669-674.

2. Frustaci A, Chimenti C, Bellocci F, Morgante E, Russo MA, MASERI A. Histological substrate of atrial biopsies in patients with lone atrial fibrillation. Circulation 1997; 96: 1180-1184.

3. Bailey GWH, Braniff BA, Hancock EW, Cohn KE. Relation of left atrial pathology to atrial fibrillation in mitral valvular disease. Ann Int Med 1968; 69: $13-20$.

4. Mary-Rabine L, Albert A, Pham TD, Hordof A, Fenoglio JJ, Malm JR, Rosen MR. The relationship of human atrial cellular electrophysiology to clinical function and ultrastructure. Circ Res 1983; 52: 188199.

5. Aimé-Sempé C, Folliguet T, Rucker-Martin C, Krajewsis S, Heimburger M, Aubier M, Mercadier J-J, ReEd JC, Hatem SN. Myocardial cell death in fibrillating and dilated human right atria. J Am Coll Cardiol 1999; 34: 1577-1586.

6. WijfFels MCEF, KirchHof CJHJ, Dorland R, Allessie MA. Atrial fibrillation begets atrial fibrillation. A study in awake chronically instrumented goats. Circulation 1995; 92: 1954-1968.

7. Morillo CA, Klein GJ, Jones DL, Whisnant JP, Holmes DR, Ilstrup DM, Frye RL. Chronic rapid 
atrial pacing: Structural, functional and electrophysiological characteristics of a new model of sustained atrial fibrillation. Circulation 1995; 91: 2968-2974.

8. Ausma J, Wijffels M, Thoné F, Wouters L, Allessie M, Borgers M. Structural changes of atrial myocardium due to sustained atrial fibrillation in the goat. Circulation 1997; 96: 3157-3163.

9. Dispersyn GD, Ausma J, Thoné F, Flameng W, Vanoverschelde J, Allessie MA, Ramaekers FCS, Borgers M. Chronic hibernating myocardium and chronic atrial fibrillation; a prelude to apoptosis? Cardiovaso Res 1999; 43: 947-957.

10. Ausma J, Wijffels M, Van Eys G, Koide M, Ramaekers F, Allessie M, Borgers M. Dedifferentiation of atrial cardiomyocytes as a result of chronic atrial fibrillation. Am J Pathol 1997; 151: 985-997.

11. Ausma J, Dispersyn GD, Duimel H, Thoné F, Ver Donck L, Allessie MA, Borgers M. Changes in ultrastructural calcium distribution in goat atria during atrial fibrillation. J Mol Cell Cardiol 2000; 32: 355-364.

12. Verbeke G, Molenberghs G. Linear mixed models in practice. A SAS-oriented approach. Springer Verlag, New York, 1993.

13. CARY NC. SAS/STAT software. Changes and enhancements through release 6.11 , SAS institute Inc. 1996.

14. Ad N, Snir E, Vidne B, Golomb E. Potential preoperative markers for the risk of developing atrial fibrillation after cardiac surgery. Semin Thora Cardiovasc Surg 1999; 11: 308-313.

15. Boyden PA, Tilley LP, Pham TD, Lui S-K, Fenoglio JJ, WIT AL. Effects of left atrial enlargement on atrial transmembrane potentials and structure in dogs with mitral valve fibrosis. Am I Cardiol 1982; 49: 1896-1908.

16. Boyden PA, Tilley LP, Albala A, Lui S-K, Fenoglio JJ, WIT AL. Mechanisms for atrial arrhythmias associated with cardiomyopathy; a study of feline hearts with primary myocardial disease. Circulation 1984: 69: 1036-1047.

17. Ausma J, Van Eys GJJM, Broers JLV, Thoné F, FlaMENG W, RAMAeKers FCS, Borgers M. Nuclear lamin expression in chronic hibernating myocardium in man. J Mol Cell Cardiol 1996; 28: 1297-1303.

18. Ausma J, Coumans W, Duimel H, Van der Vusse GJ, Allessie MA, Borgers M. Do high energy phosphates and mitochondrial enzyme activity change during prolonged atrial fibrillation? Cardiovasc Res 2000; 47: 788-796.

19. Van Gelder IC, Crijns HJGM, Blanksma PK, Landsman MLJ, Posma JL, Van den Berg MP, Meijler FL, LIE KI. Time course of hemodynamic changes and improvement of exercise tolerance after cardioversion of chronic atrial fibrillation unassociated with cardiac valve disease. Am J Cardiol 1993; 72: 560-566.

20. KNAapen MW, VRolijk BC, Wenink AC. Ultrastructural changes of the myocardium in the embryonic rat heart. Anat Rec 1997; 248: 233-241.

21. Ausma J, Schaart G, Thoné F, Shivalkar B, Flameng W, Depré C, Vanoverschelde J-L, Ramaekers F, BORGERs M. Chronic ischemic viable myocardium in man: aspects of dedifferentiation. Cardiovasc Pathol 1995; 4: 29-37.

22. SchaARt G, VAN der Ven PMF, Ramaekers FCS. Characterization of cardiotin, a structural component in the myocard. Eur J Cell Biol 1993; 62: 34-48.

23. VAN DER Loop FTL, VAN Eys GJJM, SchaART G, RaMAEKERS FCS. Titin expression as an early marker of heart and skeletal muscle differentiation in vitro: Developmental re-organization in relation to cytoskeletal constituents. J Muscle Res Cell Motil 1996; 17: 23-36.

24. Woodcock-Mitchell J, Mitchell JJ, Low RB, Kieny M, Sengel P, Rubbia L, Skalli O, Jackson B, Gabbiani G. $\alpha$-Smooth muscle actin is transiently expressed in embryonic rat cardiac and skeletal muscles. Differentiation 1988; 39: 161-166.

25. Van der Loop FTL, SchaArt G, Langmann H, RaMAEKERS FCS, ViEBAHN CH. Rearrangement of intercellular junctions and cytoskeletal proteins during rabbit myocard development. Eur I Cell Biol 1995; 68: 62-69.

26. Cary RB, Klymkowsky MW. Disruption of intermediate filament organization leads to structural defects at the intersomite junction in Xenopus myotomal muscle. Development 1995; 121: 1041-1052.

27. White CW, Kerber RE, Weiss HR, Marcus ML. The effects of atrial fibrillation on atrial pressure-volume and flow relationships. Circ Res 1982; 151: 205215.

28. Smetnev AS, Bunin I, Nargizian AB, Petrovskit PF, VAKLIAEV VD. Characteristics in the metabolism in the myocardium of patients with auricular fibrillation. Kardiologia 1983; 23: 70-73.

29. Lau CP, Leung WH, Wong CK, Cheng CH. Haemodynamics of induced atrial fibrillation: a comparative assessment with sinus rhythm, atrial and ventricular pacing. Eur Heart J 1990; 11: 219-224.

30. Jayachandran V, Winkle W, Sih HJ, Zipes DP, Olgin JE. Chronic atrial fibrillation from rapid atrial pacing is associated with reduced atrial blood flow: a positron emission tomography study. Circulation 1998; 98: I209.

31. Leistad E, Aksnes, Verburg E, Christensen G. Atrial contractile dysfunction after short-term atrial fibrillation is reduced by verapamil, but increased by BAY-K 8644. Circulation 1996; 93: 1747-1754.

32. Crijns HJGM, Wijk van LM, Gilst van WH, Kingma JH, VAN Gelder IC, Lie KI. Acute conversion of atrial fibrillation to sinus rhythm: clinical efficacy of flecainide acetate. Comparison of two regimes. Eur Heart J 1988; 9: 634-638.

33. Van der Velden HM, Ausma J, Rook M, Hellemons AjCGM, Van Veen TAB, Allessie MA, Jongsma HJ. Gap-junctional remodeling in relation to stabilization of atrial fibrillation in goats. Cardiovasc Res 2000; 46: $476-486$.

34. Ausma J, Duytschaever M, Wijffels M, Borgers M, Allessie M. Loss of efficacy of cardioversion by class Ic drugs after long term atrial fibrillation in the goat. Eur Heart J 2000; 21 Suppl: 543.

35. Todd DM, Walden AP, Fynn SP, Hobbs WJ, GarratT CJ. Repetitive one-month periods of atrial electrical remodeling promote the stability of atrial fibrillation. Circulation 2000; 102: II-154. 